\title{
Real-time and ultrafast phase retrieval in optical time- stretch using a modified Gerchberg-Saxton algorithm
}

\author{
Yiqing Xu ${ }^{1}$, Zhibo Ren ${ }^{1}$, Kenneth K. Y. Wong ${ }^{1}$, and Kevin Tsia ${ }^{1, *}$ \\ ${ }^{l}$ Department of Electrical and Electronic Engineering, The University of Hong Kong, Pokfulam Road, Hong Kong \\ *Corresponding author: tsia@hku.hk
}

\begin{abstract}
We report a new and practical scheme of using optical time-stretch with Gerchberg Saxton (GS)-like algorithm for ultrafast real-time phase retrieval, with the phase error significantly suppressed even at a wide signal bandwidth.
\end{abstract}

OCIS codes: (120.5050) Phase measurement; (350.5030) Phase.

Determining the phase of an optical signal is well-known to be the key for complete optical field characterization, which has profound impact on various applications, e.g. telecommunication, and basic science studies. Traditional techniques include nonlinear optical gating and spectral shearing interferometry for recovering the amplitude and phase of the optical field [1]. They typically require high intensity signals and are only suitable for ultrashort pulse (fs-ps) characterization. They are also limited to measure the repetitive events, and are unable to capture rapid transient behavior in the individual pulses. Notable example is ultrafast quantitative phase bioimaging, in which the spatial information of targets is not only encoded into the amplitude, but also the phase of the imaging pulses, for high-throughput or high-speed dynamical studies of the cells and tissues [2]. Other techniques exist for arbitrary optical field characterization in the longer time regime (>>ns) [3, 4]. They mostly adopted referencing methods which require external oscillator, synchronization with the signal under test and coherent detection - greatly increasing the system complexity and limiting the operation wavelength range (primarily in telecommunication band). In contrast, optical time-stretch (OTS) is an attractive tool for real-time ultrafast phase recovery of nonrepetitive optical field without coherent detection [5-6]. More specifically, this technique lies in the use of group velocity dispersion (GVD) of optical fibers to transform the spectral amplitude information of the input optical signal into temporal waveform, which can be detected in real-time continuously. High GVD is required for undistorted wavelength-to-time mapping in OTS - i.e. far-field transform. Nevertheless, one can apply GerchbergSaxton (GS)-like algorithm, with relatively low GVD (i.e. near-field transform) to numerically retrieve the phase information [6, 7]. This bypasses the need for high GVD which inevitably links to high optical loss - diminishing the detection sensitivity - yet obtains the complete field information. In this paper, we report a comprehensive study on the fundamental limitation of this technique (on phase retrieval error) and propose accordingly a modified GSlike OTS implementation to reduce the phase error even at a high signal bandwidth ( $>10 \mathrm{GHz}$ ).
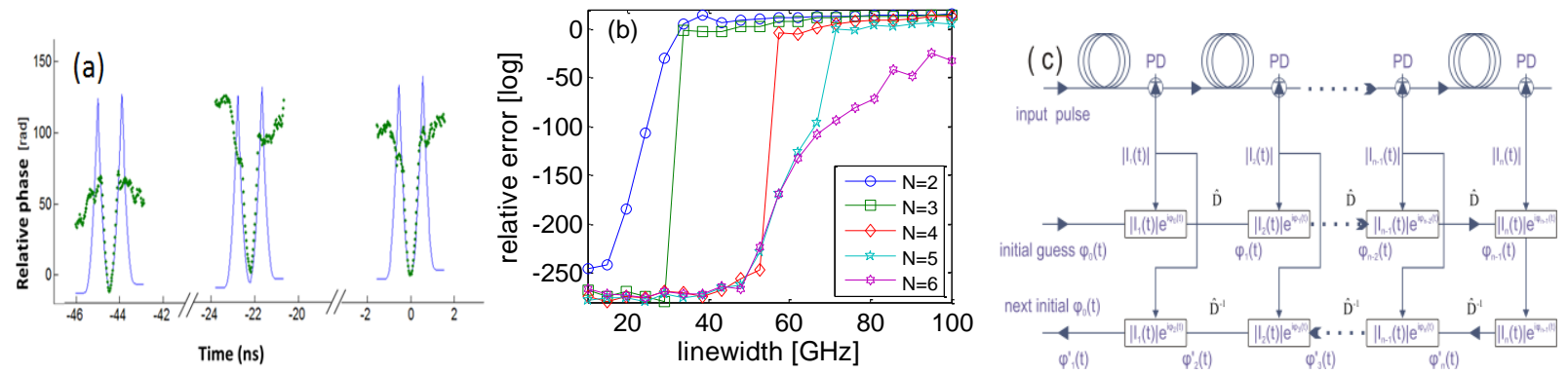

Fig. 1. (a) Experimentally retrieved optical phase of a $50 \mathrm{GHz}$ bandwidth Gaussian pulse train (from a $46 \mathrm{MHz}$ mode-locked laser with a center wavelength at $1563 \mathrm{~nm})$. Dotted curves are the phase recovered from experiment whereas solid curves are the numerically simulated phases $(N=$ 2). Two dispersion compensation fibers (DCF) with the group velocity delays of $245 \mathrm{ps}^{2}$ and $1108 \mathrm{ps}^{2}$ are employed. Note that the optical signal bandwidth is beyond the detection bandwidth $(\sim 10 \mathrm{GHz})$. (b) Phase errors as a function input signal spectral bandwidth for different intermediate steps in the newly modified GS-algorithm utilized with OTS, which is shown in (c). PD: photodetector. D is the dispersion operator.

GS algorithm is adopted here for retrieving the phase of the optical temporal signal [5,7]. It iterates and recovers the phase between two constraints - temporal and spectral intensity profiles via GVD. From the practical point of view, the detection bandwidths of the photodetector and the digitizer limit the temporal intensity information of the ultrafast input optical field. It is thus favorable to pre-time-stretch the signal as one of the "constraints" in the GS algorithm in order to relax the detection bandwidth requirement. This allows signal with a bandwidth beyond the detection limit to be recovered (see the experimental results shown in Fig. 1(a)). However, even if we assume the detection bandwidth is infinite, we found that the optical bandwidth of the signal fundamentally limits the phase retrieval accuracy in the conventional GS algorithm. To this end, we numerically simulate, by the nonlinear Schrödinger equation, a transform limited Gaussian pulse with a range of bandwidth and recover the original phase 


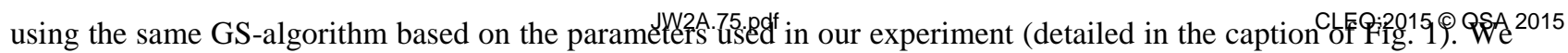
plot the recovery error as a function of the bandwidth of the input pulses in Fig. 1 (b). Clearly, the phase error of the retrieved phase increase rapidly as the optical signal bandwidth increases well beyond $50 \mathrm{GHz}$ (the case of $\mathrm{N}=2$ ). We attribute this behavior to the first DCF fiber as well as the two-step approach of the algorithm. The origin of the error can be understood as that under propagating for the same length of optical fiber, the broadening of the pulse with an exceedingly wide bandwidth becomes too profound, and drives the pulse into the far field transform regime rather than the near field one. Hence, the GS-algorithm regards it as a small transition in the intensity profile after the second DCF - resulting in a loss of the high frequency components during the iteration processes and thus it fails to converge to an accurate phase value.

To overcome this limitation, we here propose a modified GS-algorithm which further suppress the phase error in the retrieval process. The routine of the modified GS-algorithm is shown in Fig. 1 (c). Unlike iterating between the intensity profiles of the two fiber output, the modified algorithm requires additional intermediate steps of intensity measurements. In the $n^{\text {th }}$ step $\left(1<n<N\right.$, whereas $N$ is the total number of steps), the estimated phase $\varphi_{n}(t)$ is computed based on the intensity measurement $\left|I_{n}(t)\right|$ in the current $n^{\text {th }}$ step and the estimated phase at the previous step $\varphi_{n-l}(t)$ combined the measured intensity profile, i.e. $\left|I_{n}(t)\right| \exp \left[\varphi_{n-1}(t)\right]$. Each step essentially represents a segment of fiber with a known GVD. The process runs until the end of all the fiber segments, followed by the reserved operation back to the first step. The final phase in this step becomes the next initial phase of the next iteration. Our simulation shows that increasing $N$ can significantly suppress the phase recovery at a fixed signal bandwidth. More importantly, it also relaxes the optical bandwidth limitation on phase recovery accuracy (Fig. 1(b)). For $N=6$, the phase error is still maintained at a significantly low value $\sim-50 \mathrm{~dB}$ even when the signal bandwidth approaches $100 \mathrm{GHz}$. In practice, this multistage approach can simply be implemented by using fiber couplers to tap a portion of light out along a few discrete segments of dispersive fibers, and are then detected by the photodectectors simultaneously.
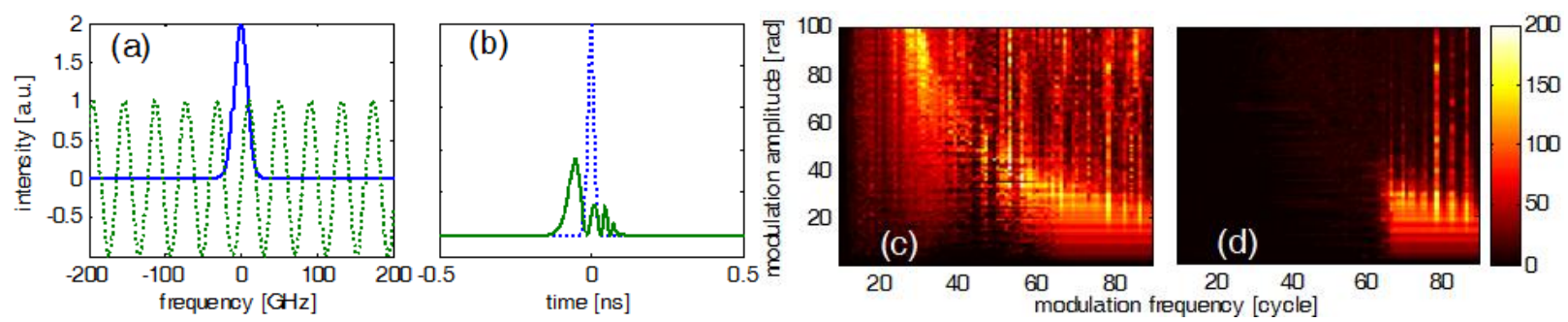

Fig. 2. (a) 10-cycle sinusoidal phase modulation (dashed) on a Gaussian input pulse spectrum (solid). (b) The temporal intensity profile after such phase modulation (solid). The input temporal profile is shown in the dotted curve. The phase error map in the case of (c) using conventional GSalgorithm with OTS and; (d) using the modified GS-algorithm ( $\mathrm{N}=6$ intermediate steps) with OTS. The color bar shows the error in a.u.

To further demonstrate the advantages of this new phase retrieval scheme with OTS, we compute the phase error map as a function of spectral phase modulation frequency and amplitude on a $20 \mathrm{GHz}$ Gaussian pulse using both convention and modified $(N=6)$ GS-algorithm. Such phase modulation on the input pulse in both spectral and temporal domain are visualized in Fig. 2(a)-(b), respectively. The significance of the error map is that it essentially indicates the phase error contribution from a range of modulation amplitude and the frequency variation. Hence, any arbitrary phase profile/perturbation can be decomposed into the bases of modulation amplitude and the frequency variation. Clearly, by comparing the two error maps, we can see that most of the low frequency and low amplitude phase modulation can be accurately retrieved by the new scheme with $N=6$ whereas the conventional GS-algorithm generally exhibits large error throughout the map. In summary, we have reported the fundamental limit of ultrafast phase recovery using OTS with conventional GS-algorithm and proposed a new scheme that can significantly reduce the phase error. This is of significant value in a broad range of applications where ultrafast continuous full optical field characterization in real-time is essential.

This work was partially supported by grant from the Research Grants Council of the Hong Kong SAR, China (Project No. HKU 7172/12E, 717510E, 717911E, 720112E, 17207714)

[1]. I. A. Walmsley and C. Dorrer, Adv. Opt. Photonics 1, 308 (2009).

[2]. A. K. S. Lau, et al., BIOMEDO 19, 076001 (2014).

[3]. Y. Park, M. Scaffardi, L. Poti, and J. Azana, Opt. Express 18, 6220 (2010).

[4]. N. K. Fontaine, et al., Nat. Photonics 4, 248 (2010).

[5]. D. R. Solli, S. Gupta, and B. Jalali, Appl. Phys. Lett. 95 (2009).

[6]. K. Goda, D. R. Solli, K. K. Tsia, and B. Jalali, Phys. Rev. A 80 (2009).

[7]. Gerchber. Rw, and W. O. Saxton, Optik 35, 237 (1972). 